\title{
Case report 483
}

\author{
Murray A. Howe, M.D. ${ }^{1}$, Kenneth A. Buckwalter, M.D. ${ }^{1}$, Ethan M. Braunstein, M.D. ${ }^{1}$, \\ and Edward M. Wojtys, M.D. ${ }^{2}$ \\ Department of ${ }^{1}$ Radiology and ${ }^{2}$ Surgery, University of Michigan Hospitals, Ann Arbor, Michigan, USA
}

\section{Imaging studies}
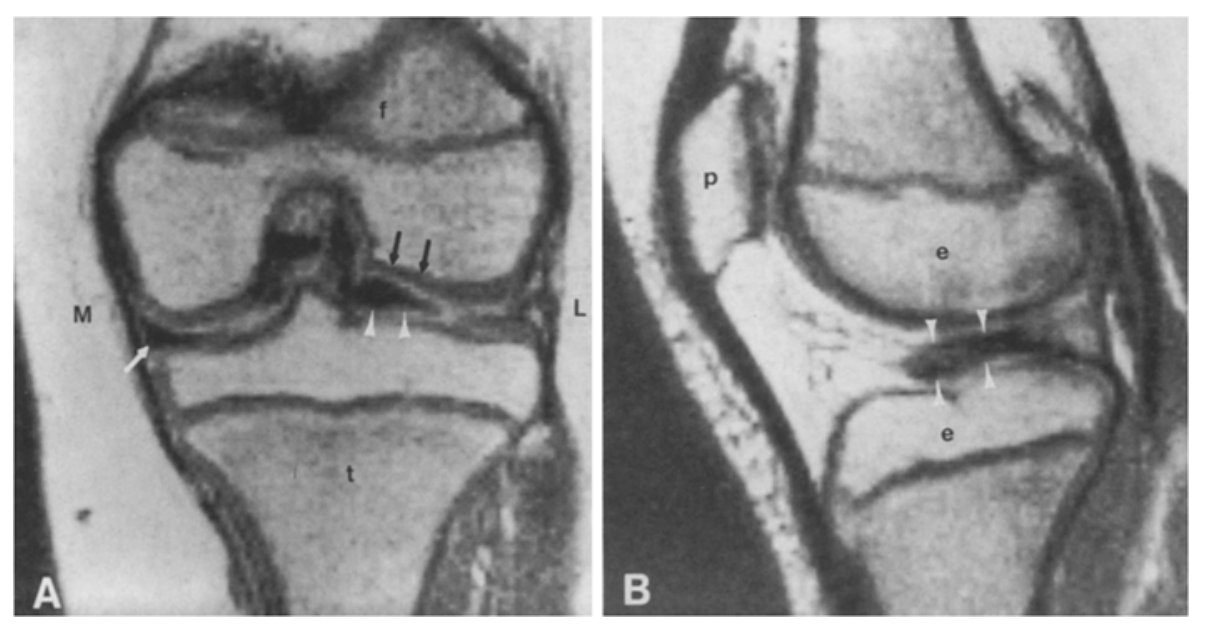

Fig. 1. A A 5-mm thick, coronal spin-echo image, midportion of the left knee, TR 2000 , TE $40 \mathrm{~ms}$. The medially displaced discoid lateral meniscus (white arrowheads) has a teardrop shape. Note the focal concavity in the adjacent medial portion of the lateral femoral condyle (black arrows). The medial meniscus (white arrows) has the expected triangular shape. $M-$ medial, $L-$ lateral, $t$ - tibia, $f$-femur. B Near midline sagittal image, TR 2000, TE $40 \mathrm{~ms}$. The central portion of the medially displaced lateral meniscus is again demonstrated (white arrowheads). $e$ - epiphysis, $p$ - patella

\section{Clinical information}

This 9-year-old girl had a 6-month history of pain in the left knee. She denied prior trauma. Blood laboratory examination was unremarkable. Physical examination revealed a small joint effusion, a click at the lateral knee joint line, and mild tenderness of the medial joint line. The examination was otherwise normal. Plain roentgenograms were normal.

Address reprint requests to: Kenneth Buckwalter, M.D., University of Michigan Hospitals, Department of Radiology, Taubman Center 2910/0326, 1500 E. Medical Center Drive, Ann Arbor, MI 48109-0326, USA
To further evaluate cartilage injury, the patient was referred for magnetic resonance imaging. Utilizing a Diasonics superconducting magnet operating at $0.35 \mathrm{~T}, 5-\mathrm{mm}$ contiguous sagittal and coronal images were acquired using spin-echo pulse sequences with a TR interval of $2000 \mathrm{~ms}$, and TE of 40 and $80 \mathrm{~ms}$. A long TR/TE was selected to optimize contrast between any potential intraarticular fluid and the articular cartilage surfaces.

On the basis of the MR findings, arthroscopy was performed. 


\section{Diagnosis: Discoid lateral meniscus (DLM), medially displaced, with complex tear}

The discoid lateral meniscus was identified on arthroscopy. Resection of the central portion of the meniscus was performed. The attempt to repair the peripheral portion of the meniscus, using an open procedure, was unsuccessful, requiring a total meniscectomy.

The MR scan shows that the lateral meniscus has an oval or teardrop shape on coronal and sagittal images (Fig. 1 A, B); it has the typical low intensity of fibrocartilage. The oval shape of the meniscus is consistent with the usual arthrographic appearance of DLM, although this was not suspected prior to the MR exam. The main substance of the meniscus is displaced medially with overgrowth of the articular cartilage at the lateral aspect of the joint. Additionally, the medial aspect of the lateral femoral condyle is concave to accommodate the abnormal meniscus. These findings suggest that the body of the meniscus has occupied a medial position for a long time, likely displaced secondary to prior injury and tear.

\section{Discussion}

Discoid lateral meniscus is an uncommon variant which has been previously described in detail [1, 2]. The usual configuration is that of an elongated, broad disc shape; however, multiple variations have been described. The etiology of DLM is uncertain. Smillie [2] has suggested that DLM represents an arrest of the normal developmental sequence; however, no discoid shape can be demonstrated in the normal maturation of mesenchymal tissue into the fibrocartilagenous meniscus. Kaplan [3] has postulated that DLM is acquired secondary to an abnormal attachment of the posterior horn to the tibial plateau. He submits that perhaps a primary abnormality of the inferior strut or fascicle leads to abnormal anterior/posterior and medial/ lateral movement of the meniscus with subsequent thickening and enlargement. This concept has been supported by further reports of poorly visualized or definitely abnormal struts in patients with DLM [3].

Due to a lack of mobile protons, fibrocartilage, ligaments, and tendons have relatively long $\mathrm{T} 1$ and short $\mathrm{T} 2$ relaxation times. Normal menisci are generally seen as homogeneously black triangular structures within the knee joint on most spin-echo pulse sequences [4]. Meniscal tears appear as intermediate intensity, intrasubstance, linear or mottled areas on T1-weighted images, and due to fluid within the tear they may demonstrate increased signal on $\mathrm{T} 2$ weighted images [5-7]. It remains unclear what the optimal pulse sequence is to evaluate these lesions, although some authors [8] propose that $\mathrm{T} 1$ or intermediate (spin-density) images are best.

DLM is well demonstrated, using both sagittal and coronal planes. Sagittal images are useful for delineating torn menisci, and coronal images may

show the anatomic extent of the DLM. Recognition of the MR appearance is helpful in preoperative evaluation.

In summary, a case of a 9-year-old girl who presented with persistent pain in the left knee was demonstrated to have a DLM. The sagittal MR images demonstrated the oval shape of the meniscus, consistent with the typical arthrographic appearance. The coronal images showed that the main substance of the meniscus was displaced medially, with overgrowth of the articular cartilage at the lateral aspect of the joint. The medial aspect of the lateral femoral condyle was concave to accommodate the abnormal meniscus. These findings are consistent with long-standing, medial displacement of the torn meniscus.

The literature concerning DLM, an uncommon variant, was reviewed and discussed.

\section{References}

1. Hall FM (1977) Arthrography of the discoid lateral meniscus. Am J Roentgenol 128:993

2. Smillie IS (1948) The congenital discoid meniscus. J Bone Joint Surg [Br] 30:671

3. Kaplan EB (1957) Discoid lateral meniscus of the knee joint. J Bone Joint Surg [Am] 39:77

4. Li KC, Henkelman RM, Poon PY, Rubenstein J (1984) MR imaging of the normal knee. J Comput Assist Tomogr 8:1147

5. Reicher MA, Hartzman S, Bassett LW, Mandelbaum B, Duckwiler G, Gold RH (1987) MR imaging of the knee. Radiology 162:547

6. Hajek PC, Baker LL, Sartoris DJ, Neumann CH, Resnick D (1987) MR arthrography: anatomic-pathologic investigation. Radiology 163:141

7. Reicher MA, Hartzman S, Duckwiler GR, Bassett LW, Anderson LJ, Gold RH (1986) Meniscal injuries : detection using MR imaging. Radiology 159:753

8. Stoller DW, Martin C, Crues JV, Kaplan L, Mink JH (1987) Meniscal tears: pathologic correlation with MR imaging. Radiology 163:731 